\title{
THE EFFECT OF MEDIA ON DOMESTIC VIOLENCE NORMS: EVIDENCE FROM INDIA
}

\section{Kuhuk Bhushan and Prakarsh Singh}

Kuhuk Bhushan is a Young Professional, Ministry of Finance, Government of India. Prakarsh Singh is a professor of economics at Amherst College, Amherst, MA USA. The corresponding author, he may be reached at psingh@amherst.edu. We are grateful to Jun Ishii, Chris Kingston, Jessica Reyes, and Yinan Zhang for helpful comments and to the Dean of the Faculty at Amherst College, Gregory Call, for funding this study.

\section{Abstract}

Greater access to media may influence norms about domestic violence. It may lead to greater acceptance of violence due to an increase in the incidence of violence or to lower acceptance due to a change in gender norms. Applying a difference-in-differences methodology to the National Family Health Survey, India (1998-99 and 2005-06), we find evidence that regularly accessing television and radio leads to a small but statistically significant reduction in the probability of women accepting domestic violence. The effect of regularly accessing both media is equivalent to the effect of three additional years of education on reducing acceptability of domestic violence. This suggests that increasing access to both media may lead to greater empowerment of women in India.

$\mathrm{P}$ hysical, sexual, and psychological violence against women is an issue of huge public health and human rights significance. The World Bank's World Development Report 2012 finds that women are at far greater risk of experiencing violence committed by an intimate partner or somebody they know than from violence by other people. And yet, 29 percent of women in a sample of 41 countries agree that wife-beating is justified for arguing with the husband, 25 percent for refusing to have sex, and 21 percent for burning food when cooking. ${ }^{1}$

Domestic violence is a global concern, and it is particularly widespread in India. While there have been many studies on the economic reasons for the persistence of gender inequalities, there has been less research on how underlying cultural beliefs on gender-based violence can be changed toward positive, nonviolent, and more peaceful outcomes. Our study adds to this literature by focusing on the use or "consumption" of media. In particular, we are interested in the interaction effects, if any, of consuming both television and radio, as possible channels by which attitudes toward domestic violence may be influenced.

\section{Pathways linking media to domestic violence attitudes} Media exposure can be a positive source for changing social norms. For example, one study on the effect of cable television on women's status in the Indian state of Tamil Nadu finds that the introduction of cable television with programs that present urban attitudes and values is associated with a 16 percent decrease in women's reported acceptance of domestic violence and an 8.8 percent decrease in their preference for giving birth to sons, as well as increases in women's autonomy and participation in household decisionmaking. ${ }^{2}$

In contrast, another study finds that better television signal reception in Indonesian villages is associated with substantially lower levels of participation in social activities, such as neighborhood associations and school committees, and lower self-reported measures of trust. This suggests that people may substitute some of the time they used to spend on social activities or household duties toward media consumption. Substitution of time away from traditional duties such as cooking or looking after children conceivably could lead to more domestic violence: In household surveys husbands often blame the wife for neglecting household duties to justify wife beating. An increase in the incidence of violence can make attitudes toward violence more accepting. Indeed, there is a large literature in psychology on the Stockholm Syndrome, where one intimate partner intermittently harasses, intimidates, threatens, beats, or otherwise abuses the other without breaking the emotional bond between them. Additionally, In India, some of the spousal violence experienced may be a male response to increasingly modern attitudes adopted by women. ${ }^{3}$

We study the relation between domestic violence norms and exposure to television and radio because despite the increasing popularity of cable television, other media such as non-cable television and radio continue to be widely accessed in India. ${ }^{4}$ The focus of media is entertainment but in contrast to television, radio has emerged as a vital source of local public information. If information is complementary to entertainment in influencing norms, we can expect interaction effects of these media sources in changing domestic violence norms. 
Similarities and differences in the content of television and radio programs may have a substitution or complementary effect on shaping attitudes toward gender and domestic violence. For instance, information about local employment or political opportunities for women, or stories about empowered women on local radio channels, may reinforce the influence of seeing empowered and independent women on television.

Using household-level data from two rounds of the National Family Health Survey (NFHS) of India, conducted in 1998-99 and 2005-06, the main finding of our study is that there is no significant effect on women's acceptance of domestic violence when they switch into either only watching television or only listening to radio in the 2005-06 period. However, the effect of television when a woman starts accessing both media regularly in the second period is associated with a 3.6 percent reduction in the probability of accepting violence (this is, after controlling for socioeconomic variables such as education of the woman, and location and wealth of the household). Strikingly, the effect is three times larger than is the effect of an additional year of education.

\section{Media in India}

Television was introduced in India in 1959, with almost all broadcasting in the hands of the state. The entry of private and foreign broadcasters in the early 1990s transformed the Indian television industry from a government-owned single network to a multichannel industry and significantly increased the amount of choice of programming for the Indian audience. The program offerings on cable television differed greatly from government programming that had primarily focused on news or information about national development. Many new soap operas introduced in the early 2000s revolved around themes of family and gender and showed women protagonists who were independent and assertive, and who often had a career or worked outside the home. These programs were an instant success and attracted very high viewership all over India. ${ }^{5}$

The public radio broadcaster, All India Radio (AIR), was established in 1936 to "inform, educate, and entertain the masses" and today has a network of 237 broadcasting centers, covering 27 languages, and reaching 99 percent of India's population. Radio has been employed as a medium for social change, such as state-supported radio rural forums for agricultural communication and promotion of adult literacy, and it continues to be an excellent source of information about social development and women's welfare.

\section{Domestic violence norms in India}

Violence against women is a major concern in India. According to a 2009 report on gender equality and women's
The article uses household-level data from two rounds of the National Family Health Survey (NFHS) of India, conducted in 1998-99 and 2005-06. The main finding is that there is no statistically significant effect on women's acceptance of domestic violence if they switch, between the first and second survey round, only to watching television or only to listening to radio. However, the effect of television when a woman starts accessing both media regularly in the second period is associated with a 3.6 percent reduced probability of accepting violence. The effect is surprisingly strong: about three times as large as is the effect of an additional year of education.

empowerment, 39 percent of currently married women aged 15-49 have experienced some kind of violence (physical, emotional, sexual) at least once in their current marriage, and 27 percent have experienced such violence in the 12 months prior to the National Family Health Survey of India, 2005-06. The most common form of emotional violence is humiliation by the husband in front of others. The most common form of physical violence is being slapped. And yet, 54 percent of women and 51 percent of men in India continue to agree with one or more of the reasons posited by the Survey for "acceptable" wife-beating. The data also suggest that violence is more likely to be deemed justified when women violate behavior expected of them in their gendered roles as wives, mothers, and daughters-in-law, such as neglecting the house or the children. ${ }^{6}$

\section{Data}

We use data from the National Family Health Survey (NFHS) of India, a large-scale multi-round survey conducted by the International Institute for Population Sciences with a representative sample of households throughout India. Three survey rounds have been conducted. We are using only those conducted in 1998-99 (NFHS-2) and in 2005-06 (NFHS-3) as detailed information on attitudes toward domestic violence is only available for these two surveys. NFHS-2 collected information from approximately 90,000 ever-married women in the age group 15-49 years. NFHS-3 expanded the scope of the survey by interviewing approximately 124,000 women of the same age group (both ever- and never-married), 109,000 households, and 74,000 men. The surveys provide information on a range of demographic, social, and economic variables such as infant and child mortality, utilization and quality of health and family planning services in the household, and indicators of household wealth, such as ownership of durable goods.

In both NFHS-2 and 3, women are asked if they believe it is justified for their husband to beat them for one or more of the following reasons: (1) if she leaves the household without 
telling him; (2) if she neglects the household or children; (3) if she does not cook food properly; and (4) if he suspects her of being unfaithful. The main outcome measure of our study captures whether a woman respondent believes that domestic violence is justified (or "acceptable") in at least one of these four situations. The NFHS surveys also provide information on households' access to media and the frequency with which these media are used. We use the frequency of accessing media as the main explanatory variable. In addition, as control variables we use the surveys' rich respondent and household characteristics such as education of the respondent and indicators of household wealth.

\section{Identification strategy}

A central empirical concern is that these empirical estimates may be statistically biased. For instance, it is possible that at any given time, women who do not believe violence is justified may be more empowered and may access more media to begin with. Correlations between media and acceptance of domestic violence might then be spurious. To address this concern, this study uses both spatial and temporal variation. Our study focuses on the relationship between changes in media access over time in each location and changes in attitudes toward violence in the same location. We employ a difference-in-differences strategy to isolate any such effect. This strategy isolates the effect of accessing media from other factors that may be correlated with both media use and attitudes toward domestic violence, such as greater economic prosperity or an increase in the levels of women's education, by controlling for baseline levels of household income and education as well as media access and also by controlling for general time trends in both media access and attitudes toward domestic violence.

Our specification also includes state fixed effects to account for fixed differences between states that may be correlated with media access and potentially can affect acceptance of domestic violence. For example, the levels of economic development and cultural norms about gender and violence may be different in different states. Also, media exposure of people living in diverse regions of India may be dissimilar due to varying levels of fluency in Hindi, the language of national broadcaster. We are able to account for these relatively stable factors through state fixed effects.

The dependent variable in this study-acceptance of domestic violence - is coded as a binary variable (it takes the value 0 if the respondent does not accept violence and 1 if she does); thus, we use both logit and probit models. The main regression specification is as follows:
(1) Accept $^{*}{ }_{\mathrm{ijt}}=\alpha_{0}+\alpha_{1}$ Post $_{\mathrm{t}}+\alpha_{2} \mathrm{TF}_{\mathrm{ijt}}+\alpha_{\mathrm{e}} \mathrm{RF}_{\mathrm{ijt}}+\alpha_{4}\left(\mathrm{TF}^{*} \mathrm{RF}\right)_{\mathrm{ijt}}$ $+\alpha_{5}\left(\text { Post }^{*} \mathrm{TF}\right)_{\mathrm{ijt}}+\alpha_{6}\left(\text { Post }^{*} \mathrm{RF}\right)_{\mathrm{ijt}}+\alpha_{7}\left(\operatorname{Post}^{*} \mathrm{TF} * \mathrm{RF}\right)_{\mathrm{ijt}}+\mathbf{X}_{\mathrm{ijt}} \beta$ $+\gamma_{\mathrm{j}}+\epsilon_{\mathrm{ijt}}$.

Accept ${ }^{*}$ ijt is a measure denoting the probability that respondent $i$ in state $j$ at time $t$ accepts domestic violence for at least one reason. As this probability is not directly observable, we use the actual acceptance of violence, Accept $_{i j t}$, to estimate the association between explanatory variables and the probability of accepting violence. The explanatory variables are as follows:

- Post is a time dummy (0 for 1998-99; 1 for 2005-06);

- $T F_{i j t}$ is a dummy variable for whether the respondent watches television at least once a week ( 0 if no; 1 if yes);

- $R F_{i j t}$ is a dummy variable for whether the respondent listens to the radio at least once a week ( 0 if no; 1 if yes);

- $\boldsymbol{X}_{i j t}$ are respondent and respondent's household characteristics that may be correlated with the frequency of accessing media and acceptance of violence; we control for respondents' education and occupation, location of the household (rural or urban), and wealth, religion, and caste of the household;

- $\gamma_{\mathrm{j}}$ are state fixed effects.

Errors are clustered at the state level to account for serial correlation between standard errors. The binary logit model estimates the effect of each of the explanatory variables on the probability of outcome Accept $_{i j t}=1$, i.e., the acceptance of violence. A positive sign for a coefficient indicates that a particular variable is correlated with an increase in the probability of accepting violence.

The coefficients $\alpha_{2}, \alpha_{3}$, and $\alpha_{4}$ are the differences at baseline between respondents who access media frequently and those who do not. For example, the probability of accepting violence is higher by $\alpha_{2}$ (or lower, if $\alpha_{2}$ is negative) for a respondent at baseline who watches television regularly but does not access radio regularly compared to a respondent who does not access either medium regularly.

A positive sign for the $\alpha_{7}$ coefficient suggests that, in the 2005-06 period, women believe it is more acceptable for their husbands to beat them if they spend time accessing both television and radio. In contrast, a negative sign would indicate a net complementary effect between accessing both media leading to a decrease in the probability of acceptance of violence by women.

Main results

Table 1 reports the estimations for the main specification. 
Columns (1) and (3) show the results for the logit and probit models, respectively, and columns (2) and (4) report the marginal effects. (Column (5) shows OLS results by way of comparison.)

We find that the direct effects of only watching television or only listening to radio are statistically insignificant (the $T F$ and $R F$ lines in Table 1), as are all the interaction effects except that for accessing both television and radio (the Post*TF*RF line). The latter effect is associated in a statistically significant way with a 3.6 percent reduction in the probability of the acceptance of violence [columns (2) and (4)]. This suggests a complementary effect of television and radio on the probability of women accepting domestic violence.

In addition, the level of education of the respondent and rural location of the household are statistically significant.. A small increase in education, say by one year, is associated with a 1.3 percent decrease in the probability of accepting violence. Rural location is associated with 5.2 percent higher likelihood of a woman accepting violence from her husband. This result suggests that greater education of women can be a particularly powerful force in changing attitudes toward domestic violence and women's status in society. It is noteworthy that exposure to both television and radio equals the effect of about three additional years of education on reducing acceptability of domestic violence.

\section{Subsidiary results}

The conceptual framework suggests two channels through which access to media could affect acceptance of violence. The first suggests that media access may positively influence norms about women's status in society and hence reduce women's acceptance of violence. In contrast, the second channel posits that greater access to media may mean that women spend more time away from household duties, making them more likely to experience, and thus to accept, violence. Our findings suggest that the first channel dominates.

To investigate the first channel, we analyze the effect of media access on the variable money (whether the woman has a say in how to spend household income). Column (1) of Table 2 finds that while over time women have greater say in how to spend household income, there is no statistically significant association of this with switching into accessing either television or radio over time.

To check for the theoretical possibility of an increase in the acceptability of violence following an increase in access to media, we analyze changes by individual reasons for accepting violence. The individual reasons for accepting violence are: (1) Go (the wife leaves the house without telling the husband); (2) Neglect (she neglects the house or children); (3) Cook (she does not cook properly); and (4) Not faithful (he suspects her of being unfaithful). It can be argued that cooking and taking care of the household are more time intensive and may be adversely affected by watching television or listening to radio. But suspicion of being unfaithful and leaving the house without 
telling the husband do not involve a cost imposed by access to media. Thus, if the second channel of an increase in violence acceptance due to media access has explanatory power, the difference-in-differences coefficients for Neglect and Cook are expected to be positive as opposed to Go and Unfaithful, which would be expected to remain negative as in the baseline regression.

Columns (2) to (5) of Table 2 show that switching into accessing either only television or only radio (the $T F$ and $R F$ lines) does not have any statistically significant effect on the probability of accepting violence for any reason. The complementary effect of accessing television and radio on acceptance of violence is significant for the reasons Go and Not faithful, but not for Neglect and Cook. This suggests that although the second channel cannot be rejected as the coefficients for Neglect and Cook regressions are statistically insignificant and negative, the evidence for time substitution driving a change in norms through an increase in violence appears to be relatively weak.

A change in the incidence of violence due to greater media access may lead to a change in the acceptance of violence by women. We use the variable Violence, defined as whether a woman has experienced domestic violence, as the dependent variable in the main specification. Column (6) shows that for women who switch into only listening to radio, there is no correlation between probability of experiencing domestic violence and listening to radio. In contrast, women who switch into watching only television are 5.6 percent more likely to experience domestic violence. This indicates that there may be some substitution between household duties and watching television that increases the probability of experiencing violence, or women who watch TV may have become more outspoken and this makes their husbands react with more violence. In contrast, when women switch into watching both media, the interactive effect between the media is associated with a 4.9 percent decrease in the probability of being beaten, consistent with the complementary effect between television and radio found in the main result (Table 1). This could be either because accessing both media leads to a change in gender norms, or because women who access both media allocate their hours between household duties and accessing media differently from women who access only one medium.
The lack of detailed variables on gender norms and information on the amount of time respondents spend on each of these activities limits our ability to explore this possibility further. ${ }^{7}$

\section{Policy}

Violence has large economic costs, including the loss of women's labor hours and productivity. The average cost per incidence of violence, according to one paper, was about one third of the monthly household income in slum and rural communities. $^{8}$

Our study examines two channels through which media access could have affect acceptance of violence: Accessing media could positively influence norms about women's status and reduce acceptance of violence or it may lead to substitution away from household duties, which may then increase the probability of experiencing and accepting violence. Using household-level data on media use and acceptance of violence by women, we find evidence that regularly accessing both television and radio has, over time, a small but statistically significant effect on reducing the probability of women accepting violence. This association holds only when women 
access both media regularly, indicating a complementary effect of accessing television and radio in reducing acceptance of violence. We do not find strong evidence for the substitution hypothesis. Strikingly, the effect of regular access to both television and radio on reducing the acceptability of domestic violence is statistically equivalent to having three additional years of education. This shows that in cases where educational attainment is costly, either because of lack of schools or due to high opportunity costs for adults, nudges toward improving access to both radio and television can increase the voice of women within the household.

The results of our analyses are but a first step toward informing policymakers on the most effective ways of imparting information to empower women in India. State policies that subsidize and encourage the use of complementary media together may help to bring about a change in women's acceptance of domestic violence and perhaps also influence other norms about gender and women's status in society. Future studies may delve into understanding the opportunity cost of accessing different media and advance our knowledge about how the content of media and its duration may affect domestic violence norms.

\section{Notes}

1. See the World Development Report 2012 on Gender Equality and Development for additional information.

\section{Jensen and Oster (2009).}

3. In contrast: Olken (2009). Wife beating: See IIPS (2009). Stockholm Syndrome: See Dutton and Painter (1981). Modern attitudes: Simister and Mehta (2010).

4. The three most popular radio stations attracted about 40 million listeners collectively in 2008, a reach comparable to that of the press and private television channels (Ranganathan and Rodrigues, 2010).

5. See Ranganathan and Rodrigues (2010) and Munshi (2010) for more details.

6. IIPS (2009).

7. See Bhushan and Singh (2013) for heterogeneous effects by literacy and geographical location, as well as robustness checks.

8. Burton, et al. (2000).

\section{References}

Bhushan, K. and P. Singh. 2013. "Impact of Media on Domestic Violence Norms: Evidence from India." Available at SSRN: http://ssrn.com/abstract=2332901

Burton, B., N. Duvvury, and N. Varia. 2000. "Domestic Violence in India, Part 3: A Summary Report of a Multi-Site Household Survey." Washington, D.C.: International Center for Research on Women.
Dutton, D.G and S.L. Painter. 1981. "Traumatic Bonding: The Development of Emotional Attachments in Battered Women and other Relationships of Intermittent Abuse." Victimology: An International Journal. Vol. 1, No. 4, pp. 139-155.

[IIPS] International Institute for Population Sciences. 2009. Gender Equality and Women's Empowerment in India. Mumbai: IIPS.

Jensen, R. and E. Oster. 2009. "The Power of TV: Cable Television and Women's Status in India." Quarterly Journal of Economics. Vol. 124, No. 3, pp. 1057-1094. http://dx.doi.org/10.1162/qjec.2009.124.3.1057

Munshi, S. 2010. Prime Time Soap Operas on Indian Television. New Delhi: Routledge.

Neurath, P.M. 1962. "Radio Farm Forum as a Tool of Change in Indian Villages." Economic Development and Cultural Change. Vol. 10, No. 3, pp. 275-283. http://dx.doi.org/10.1086/449962

Olenick, W. 2000. "Women's Exposure to Mass Media Is Linked to Attitudes Toward Contraception in Pakistan, India and Bangladesh." International Family Planning Perspectives. Vol. 26, No. 1, pp. 48-50. http://dx.doi.org/10.2307/2648294

Olken, B. A. 2009. "Do Television and Radio Destroy Social Capital? Evidence from Indonesian Villages." American Economic Journal: Applied Economics. Vol. 1, No. 4, pp. $1-33$.

Ranganathan, M. and U. Rodrigues. 2010. Indian Media in a Globalised World. New Delhi: SAGE Publications. http://dx.doi.org/10.4135/9788132105992

Simister, J. and P.S. Mehta. 2010. "Gender Based Violence in India: Long-Term Trends." Journal of Interpersonal Violence. Vol. 25, No. 9, pp. 1594-1611. http://dx.doi.org/10.1177/0886260509354577

[WHO] World Health Organization. 2005. WHO Multi-country Study on Women's Health and Domestic Violence Against Women. Geneva: WHO.

World Bank. 2012. The World Development Report 2012: Gender Equality and Development. Washington, D.C.: World Bank. 